\title{
Dynorphin-Dependent Reduction of Excitability and Attenuation of Inhibitory Afferents of NPS Neurons in the Pericoerulear Region of Mice
}

\author{
Kay Jüngling *, Peter Blaesse, Lena Goedecke and Hans-Christian Pape \\ Institute of Physiology I, University of Münster, Münster, Germany
}

The Neuropeptide S system, consisting of the 20-amino acid peptide neuropeptide S (NPS) and its G-protein coupled receptor (NPSR), modulates arousal, wakefulness, anxiety, and fear-extinction in mice. In addition, recent evidence indicates that the NPS system attenuates stress-dependent impairment of fear extinction, and that NPS-expressing neurons in close proximity to the locus coeruleus region (LC; pericoerulear, periLC) are activated by stress. Furthermore, periLC NPS neurons receive afferents from neurons of the centrolateral nucleus of the amygdala (CeL), of which a substantial population expresses the kappa opioid receptor (KOR) ligand precursor prodynorphin. This study aims to identify the effect of the dynorphinergic system on NPS neurons in the periLC via pre- and postsynaptic mechanisms. Using electrophysiological recordings in mouse brain slices, we provide evidence that NPS neurons in the

OPEN ACCESS

Edited by:

Gerald W. Zamponi,

University of Calgary, Canada

Reviewed by:

Annalisa Scimemi,

The State University of New York at

Albany, USA

Terence Hébert,

McGill University, Canada

*Correspondence:

Kay Jüngling

kay.juengling@gmx.de

Received: 28 January 2016 Accepted: 25 February 2016

Published: 11 March 2016

Citation:

Jüngling K, Blaesse P, Goedecke L and Pape H-C (2016)

Dynorphin-Dependent Reduction of

Excitability and Attenuation of Inhibitory Afferents of NPS Neurons in the Pericoerulear Region of Mice.

Front. Cell. Neurosci. 10:61.

doi: 10.3389/fncel.2016.00061 periLC region are directly inhibited by dynorphin A (DynA) via activation of $\kappa$-opioid receptor 1 (KOR1) and a subsequent increase of potassium conductances. Thus, the dynorphinergic system is suited to inactivate NPS neurons in the periLC. In addition to this direct, somatic effect, DynA reduces the efficacy of GABAergic synapses on NPS neurons via KOR1 and KOR2. In conclusion, the present study provides evidence for the interaction of the NPS and the kappa opioid system in the periLC. Therefore, the endogenous opioid dynorphin is suited to inhibit NPS neurons with a subsequent decrease in NPS release in putative target regions leading to a variety of physiological consequences such as increased anxiety or vulnerability to stress exposure.

Keywords: neuropeptide S, kappa opioid receptor, anxiety, stress, synaptic transmission

\section{INTRODUCTION}

The neuropeptide S (NPS) system, consisting of the 20-amino acid peptide NPS and its G-protein coupled receptor (NPSR), has been shown to be involved in processes of anxiety, fear-extinction, and fear memory consolidation (Xu et al., 2004; Okamura and Reinscheid, 2007; Jüngling et al., 2008; Okamura et al., 2011). Recent evidence suggests that the NPS system is involved in stress coping and that stressful events increase the release of NPS in the amygdala of rodents (Ebner et al., 2011; Chauveau et al., 2012). In line with this, immobilization stress induces an up-regulation of c-fos in NPS-expressing neurons, indicating an activation of these neurons during periods of stress (Liu et al., 2011; Jüngling et al., 2012). 
The NPS neurons express the corticotropin-releasing factor receptor 1 (CRF1) and depolarize following CRF1 activation by CRF or stressin I (Liu et al., 2011; Jüngling et al., 2012).

Stressful conditions additionally promote the activation of endogenous opioid systems (for review, Knoll and Carlezon, 2010) and the release of dynorphin A (DynA), which in turn activates $\mathrm{K}$-opioid receptors (KOR; Chavkin et al., 1982). Activation of the dynorphin system induces analgesia, but also aversive and prodepressive-like behavior in rodents (Mague et al., 2003; Carlezon et al., 2006; Bruchas et al., 2007; Land et al., 2008, 2009; Knoll and Carlezon, 2010). The activation of KORs has been shown to modulate the excitability of neurons and directly influence transmitter release by presynaptic mechanisms. Depending on the cell type and brain region, dynorphin can either increase or decrease the activity of neurons. An increase in activity can be based on e.g., the enhancement of a hyperpolarization-activated current $\left(I_{h}\right.$; Pan, 2003) or on the modulation of voltage-dependent potassium currents (McDermott and Schrader, 2011). In contrast, a decrease in neuronal activity can be mediated by an enhancement of potassium conductances, most likely inwardly rectifying potassium conductances (Grudt and Williams, 1993, 1995; Ma et al., 1995). In rodents, agonists of KORs attenuate the release of various neurotransmitters and neuropeptides on the norepinephrine neurons of the locus coeruleus (LC) by presynaptic inhibition (Kreibich et al., 2008). Neurons of the $\mathrm{LC}$ receive afferents from the central amygdala (Dimitrov et al., 2013) and these afferents can contain CRF and/or dynorphin (Reyes et al., 2008, 2011). NPS-expressing neurons in the periLC region have recently been shown to receive central amygdalar afferents originating from dynorphin- and somatostatin-expressing neurons (Jüngling et al., 2015). In addition, NPS neurons are inactivated by application of DynA or somatostatin, indicating a negative control of NPSexpressing neurons by these peptide systems (Jüngling et al., 2015).

Based on the findings that the NPS system is involved in stress responses and that NPS neurons in the periLC respond to $\mathrm{CRF}$ and DynA, two key players of stress responses, we used in vitro slice electrophysiology to analyze the effects of DynA on NPS neurons in more detail and to identify the underlying synaptic mechanisms. In addition to the direct inhibitory effect of DynA on NPS neurons, we analyzed its role in the regulation of synaptic efficacy of GABAergic afferents projecting onto NPS neurons.

\section{MATERIALS AND METHODS}

\section{Animals}

Heterozygous transgenic mice expressing the enhanced green fluorescent protein (EGFP) under the control of the NPS promotor (transgenic NPS-EGFP mouse line E16; Liu et al., 2011) were bred with $\mathrm{C} 57 \mathrm{Bl} / 6$ mice and offspring was genotyped by PCR as described previously (Liu et al., 2011). Mice were kept in a temperature $\left(21^{\circ} \mathrm{C}\right)$ and humidity-controlled (50-60\% relative humidity) animal facility with access to food and water ad libitum and a 12:12 h light-dark cycle with lights on at 6:00 AM. All animal experiments were carried out in accordance with national regulations on animal experimentation (European Committees Council Directive 86/609/EEC; National Research Council of the National Academies) and protocols were approved by the local authorities (Bezirksregierung Münster, AZ 50.0835.1.0, G 53/2005) and "Landesamt für Natur, Umwelt und Verbraucherschutz Nordrhein-Westfalen" (reference number: 8.87-51.05.20.10.218).

\section{Electrophysiology}

Six to ten weeks old NPS-EGFP mice of either sex were anesthetized with isoflurane (Forene, 1-Chloro-2,2, 2-trifluoroethyl-difluoromethylether; 2.5\%) and killed by decapitation. Horizontal slices (300 $\mu \mathrm{m}$ thick) containing the LC were prepared. Whole-cell patch-clamp recordings (in voltage- or current-clamp mode) were performed as described previously (Jüngling et al., 2008). We used patch pipettes made of borosilicate glass (GC150T-10, Harvard Apparatus, Edenbridge), pulled on a vertical puller (PA-10, E.S.F. Electronic, Germany). The intracellular solution used to analyze the intrinsic properties of NPS-EGFP neurons contained [in $\mathrm{mM}$ ]: $\mathrm{NaCl} 10, \mathrm{~K}$-gluconate $105, \mathrm{~K}_{3}$-citrate 20, HEPES 10, BAPTA 3, $\mathrm{MgCl}_{2} 1, \mathrm{MgATP} 3$, and NaGTP 0.5. The $\mathrm{pH}$ was adjusted to 7.25. Artificial cerebrospinal fluid (aCSF) was used as extracellular solution and contained [in mM]: $\mathrm{NaCl} 120, \mathrm{KCl} 2.5, \mathrm{NaH}_{2} \mathrm{PO}_{4} 1.25, \mathrm{MgSO}_{4} 2, \mathrm{CaCl}_{2}$ 2 , and glucose 20 . The $\mathrm{pH}$ was adjusted to 7.3 by gassing with carbogen $\left(95 \% \mathrm{O}_{2}, 5 \% \mathrm{CO}_{2}\right)$. The liquid junction potential was corrected for $(10 \mathrm{mV})$. The pipette resistance was between 2.2 and 2.8 $\mathrm{M} \Omega$ using the intracellular solution listed above. The series resistance RS was between 5-15 M $\Omega$ and recordings with higher RS were rejected from analysis. All experiments were performed at $30-32^{\circ} \mathrm{C}$.

Active and passive membrane properties were assessed during whole-cell current-clamp recordings at a membrane potential of $-60 \mathrm{mV}$. Hyper- and depolarizing currents were injected for $500 \mathrm{~ms}$ (injected currents from -80 to $+140 \mathrm{pA} ; \Delta \mathrm{I}+10 \mathrm{pA}$ ). Active membrane properties were analyzed during depolarizing current injections of +80 to $+120 \mathrm{pA}$. The input resistance of the recorded neurons was calculated by $\mathrm{R}_{\text {input }}-\Delta \mathrm{V} / \mathrm{I} . \Delta \mathrm{V}$ was determined under steady-state conditions at the end of an injected hyperpolarizing current pulse $(\mathrm{I}=-50 \mathrm{pA})$. The membrane time constant $\tau$ was obtained by a monoexponential fit of the membrane potential shift. The resting membrane potential was measured immediately after establishing the wholecell configuration. The after-hyperpolarizing potential (AHP) was determined after the first action potential (AP). The slow AHP (sAHP) was defined as hyperpolarizing voltage deflection following a $500 \mathrm{~ms}$ current injection of $+80 \mathrm{pA}$. The AP halfwidth was extracted from the first AP. The time-to-first-AP was calculated as time from the beginning of the depolarizing current injection $(+80 \mathrm{pA})$ to the onset of the first AP. The instantaneous frequency was calculated between the first two APs occurring in response to a depolarizing current injection of $+80 \mathrm{pA}$.

Electrophysiological data were acquired with an EPC10-double amplifier (HEKA, Germany) at a sampling 
rate of $10 \mathrm{kHz}$ and analyzed offline with Clampfit10 software (Molecular Devices Corporation, Sunnyvale, CA, USA).

\section{Drug Testing}

To analyze the effect of opioid receptor agonists, NPS-EGFP neurons were recorded either in the current-clamp mode at a membrane potential around $-65 \mathrm{mV}$ or in the voltage-clamp mode at $-65 \mathrm{mV}$. Drugs were bath-applied for $5 \mathrm{~min}$ at a perfusion speed of $\sim 3.5 \mathrm{ml} / \mathrm{min}$. In the current-clamp mode, the input resistance was monitored by injecting hyperpolarizing currents of $-50 \mathrm{pA}$. During maximal drug effect, the membrane potential was manually set to baseline values by adjusting a DC offset to exclude changes of the input resistance induced by voltage-dependent conductances. Gabazine (GBZ; $25 \mu \mathrm{m})$ or picrotoxin $(100 \mu \mathrm{m})$, CGP55845 $(10 \mu \mathrm{m})$, D-(-)-2-Amino-5-phosphonopentanoic acid (AP5, $50 \mu \mathrm{m}$ ), and 6,7-Dinitroquinoxaline-2,3-dione (DNQX, $10 \mu \mathrm{m}$ ) were added to the bathing solution as required to block GABAergic and glutamatergic postsynaptic currents, respectively. In some experiments, tetrodotoxin (TTX, $0.5-1 \mu \mathrm{m}$ ) was added to decrease network activity (toxins were purchased from Biozol Diagnostica, Germany).

The KOR agonist DynA, the $\mu$-opioid receptor (MOR) agonist [D-Ala2, N-MePhe4, Gly-ol]-enkephalin (DAMGO), and the $\delta$-opioid receptor (DOR) agonist SNC-80 were applied at a concentration of $500 \mathrm{nM}$ (all from Tocris). The KOR antagonist GNTI (5'-Guanidinyl-17(cyclopropylmethyl)-6,7-dehydro-4,5 $\alpha$-epoxy-3,14-dihydroxy-6, $7-2^{\prime}, 3^{\prime}$-indolomorphinan dihydrochloride; Tocris) was applied prior to DynA at a concentration of $1.5 \mu \mathrm{m}$. The К-opioid receptor 1 (KOR1) agonist U69,593((+)- $(5 \alpha, 7 \alpha, 8 \beta)$ N-Methyl-N-[7-(1-pyrrolidinyl)-1-oxaspiro[4.5]dec-8-yl]-benze neacetamide; Sigma Aldrich) and the KOR2 agonist GR 89696 fumarate (4-[(3,4-Dichlorophenyl)acetyl]-3-(1-pyrroli dinylmethyl)-1-piperazinecarboxylic acid methyl ester fumarate, Tocris) were bath-applied at a concentration of $250 \mathrm{nM}$. For co-application of DynA and CRF (Tocris), both drugs were used at a concentration of $250 \mathrm{nM}$. The CRF receptor 1 (CRF1) antagonist antalarmin hydrochloride (Tocris) was used at a concentration of $4 \mu \mathrm{m}$. Tertiapin Q (Tocris), an antagonist of ROMK1 and GIRK1/4 potassium channels, was bath-applied at a concentration of $200 \mathrm{nM}$. In some experiments, $0.5 \mu \mathrm{m} \mathrm{BaCl} 2$ was added to the aCSF. When using $\mathrm{BaCl}_{2}, \mathrm{MgSO}_{4}$ was replaced by $1.5 \mathrm{mM} \mathrm{MgCl}_{2}$ in the aCSF.

\section{Voltage-Clamp Ramps}

To analyze the nature of substance-induced currents, depolarizing voltage-clamp ramps were performed (from -120 to $-20 \mathrm{mV} ; 3 \mathrm{~s}$ ) from a holding potential of $-60 \mathrm{mV}$. The recordings were done in presence of $1 \mu \mathrm{m}$ TTX to reduce network activity and $100 \mu \mathrm{m} \mathrm{CdCl} 2$ to reduce calcium currents during depolarizing ramps. Ramps were recorded during baseline conditions (Rampbaseline) and during maximal drug effect (Rampdrug). The resultant, substance-induced current was calculated by Ramp drug - Ramp baseline and plotted vs. the holding potential.

\section{Evoked Inhibitory Postsynaptic Currents (eIPSCs)}

To evoke $\mathrm{GABA}_{\mathrm{A}}$ receptor $\left(\mathrm{GABA}_{\mathrm{A}} \mathrm{R}\right)$-mediated postsynaptic currents in NPS neurons, a tungsten bipolar stimulation electrode was placed rostral to the NPS neurons in the periLC. NPS neurons were recorded in voltage-clamp mode at a holding potential of $-65 \mathrm{mV}$ using a KCl-based intracellular solution. Recordings were done in presence of AMPA, NMDA and $\mathrm{GABA}_{\mathrm{B}}$ receptor antagonists. The latency was measured between the stimulation artifact and the onset of the postsynaptic responses. Responses with latencies $<5 \mathrm{~ms}$ were accepted for analysis. Evoked inhibitory postsynaptic currents (eIPSCs) were considered as failures when it lacked typical IPSC kinetics and when the amplitude was smaller than two times the standard deviation of the mean baseline noise. Brief (50-100 $\mu$ s) electrical pulses were delivered with an interstimulus interval of $20 \mathrm{~s}$. The stimulation strength was set to allow failures in the majority of recordings during baseline stimulation.

\section{Miniature Inhibitory Postsynaptic Currents (mIPSCs)}

The miniature IPSCs (mIPSCs) were recorded in the voltageclamp mode at a holding potential of $-65 \mathrm{mV}$ using a high chloride intracellular solution, containing $105 \mathrm{mM} \mathrm{KCl}$ instead of $\mathrm{K}$-gluconate. To block AP triggered release, $0.5 \mu \mathrm{m}$ tetrodotoxin was added to the aCSF. DNQX, AP-5, and CGP55845 were added to the aCSF to antagonize AMPA-, NMDA-, and $\mathrm{GABA}_{B}$ receptor-mediated currents, respectively, and to record pharmacologically isolated $\mathrm{GABA}_{\mathrm{A}} \mathrm{R}$-mediated mIPSCs. Neurons were allowed to equilibrate for at least $5 \mathrm{~min}$ prior to recordings. Frequency, amplitudes and kinetics of mIPSCs, rise time $_{10-90 \%}$ and decay time $e_{90-10 \%}$, were automatically analyzed during baseline conditions and in presence of DynA using Clampfit10.

\section{Statistics}

Data are represented as box plots, in which the box represents the first $(25 \%)$ and third $(75 \%)$ quartiles, the band represents the median, and the whiskers represent the percentiles 5\% and 95\%. The square within the box represents the mean. All data sets were tested for statistically significant outliers using the Grubbs' test (significance level $p<0.05$ ). Within group comparisons were done by using a paired student's $t$-test (significance level ${ }^{*} p<0.05 ;{ }^{* *} p<0.01$ ). To analyze differences between different groups, one-way ANOVA followed by Bonferroni post hoc test or unpaired student's $t$-test were used (significance level ${ }^{*} p<0.05$; $\left.{ }^{* *} p<0.01\right)$.

\section{RESULTS}

Gene expression profiling of isolated NPS neurons from the periLC of NPS-EGFP mice indicates the expression of KORs in these neurons (Liu et al., 2011). Furthermore, it has previously 

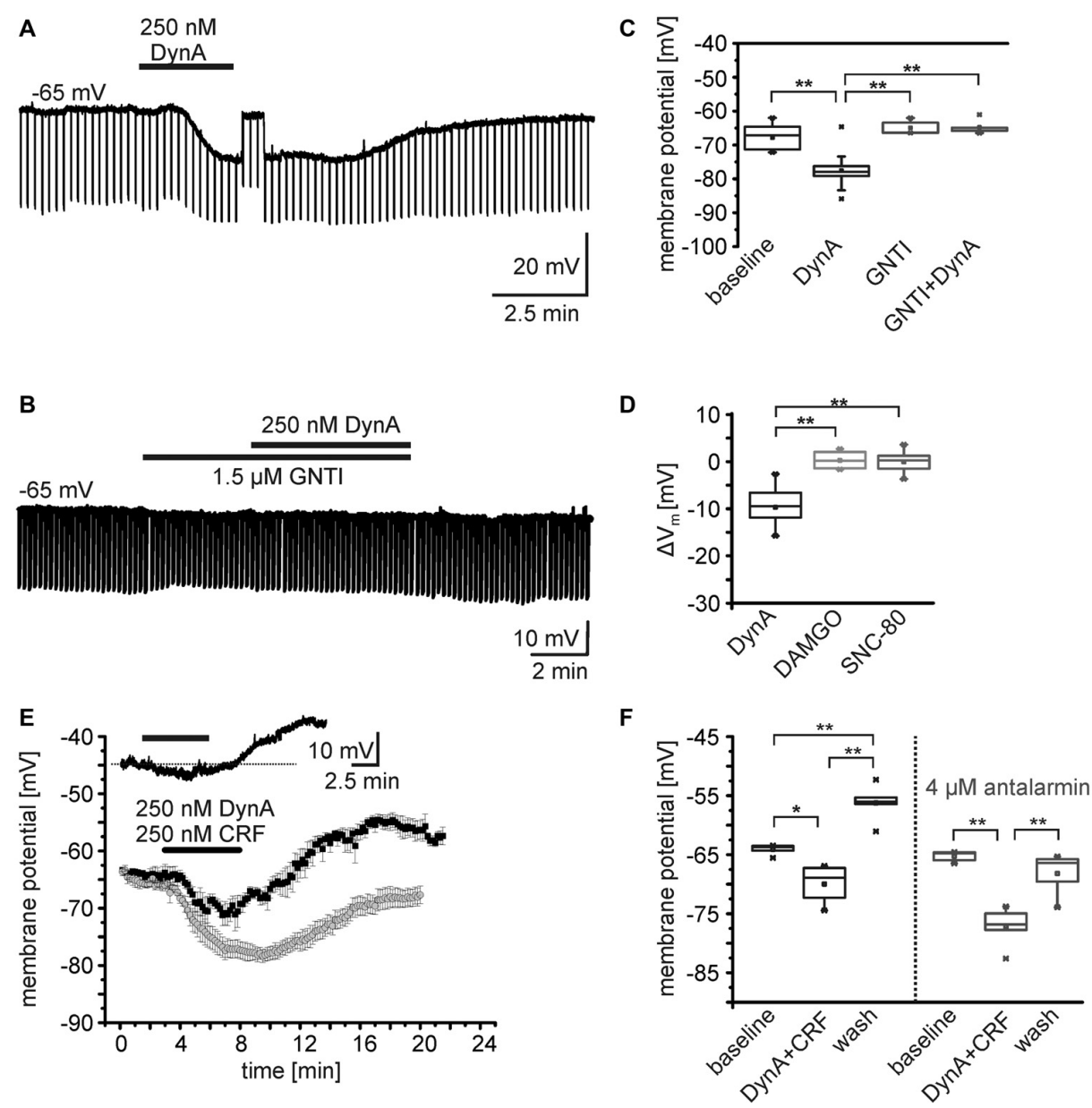

FIGURE 1 | Effects of opioid receptor agonists on the membrane potential of NPS neurons. (A,B) Sample current-clamp recordings of NPS neurons in the periLC region. Hyperpolarizing current pulses (-50 pA; $500 \mathrm{~ms}$ ) were applied to monitor the input resistance of the recorded neuron. While application of $250 \mathrm{nM}$ DynA induced a transient hyperpolarization (A), the kappa opioid receptor (KOR) antagonist GNTI hydrochloride prevented the DynA effect (B). To monitor drug-induced changes in input resistance, the membrane potential was manually set to baseline values by adjusting a DC offset during maximal drug effect (transient jump in membrane potential seen in A). This allows distinguishing between direct drug-induced changes in input resistance and indirect changes induced by voltage-dependent conductances. (C) Quantification of the effect of DynA on the membrane potential in the absence ( $n=14$ ) or presence of GNTI hydrochloride $(n=5)$. (D) Quantification of the mean membrane potential shift following application of $250 \mathrm{nM}$ DynA $(n=14), 500 \mathrm{nM}$ DAMGO $(n=9)$ and $500 \mathrm{nM}$ SNC-80 $(n=9)$ (E) Time course of the membrane potential changes in response to DynA/CRF co-application in absence (black) or presence (gray) of the corticotropin-releasing factor receptor 1 (CRF1) antagonist antalarmin. Inset shows a sample recording in current-clamp mode. (F) Quantification of the membrane potential changes in NPS neurons during DynA/CRF co-application $(n=5)$ and DynA/CRF co-application in presence of antalarmin $(n=5)$.

been shown that the neuropeptides DynA and somatostatin hyperpolarize NPS neurons in the periLC region (Jüngling et al., 2015). To reveal detailed information on the underlying cellular mechanisms and the specificity of the DynA application and the subsequent activation of KORs, EGFP-expressing NPS neurons (here referred to as NPS neurons) were recorded in the whole-cell current-clamp mode at a membrane potential set around $-65 \mathrm{mV}$. In the presence of glutamate and GABA receptor antagonists, and $0.5 \mu \mathrm{m}$ TTX, the application of $250 \mathrm{nM}$ DynA induced a significant membrane hyperpolarization in all neurons tested ( $n=14$; Figures 1A,C). In agreement with previous results, the average membrane potential $\left(\mathrm{V}_{\mathrm{m}}\right)$ of NPS neurons during baseline conditions was at $-67.9 \pm 1.1 \mathrm{mV}$ and shifted to $-77.6 \pm 1.4 \mathrm{mV}$ in the presence of DynA
$\left(\Delta \mathrm{V}_{\mathrm{m}}=-9.5 \pm 1.0 \mathrm{mV} ; p=6.806 \mathrm{E}-7 ; t=9.42 ; \mathrm{DoF}=12 ;\right.$ Figure 1D). The DynA-induced hyperpolarization was absent in the presence of the KOR antagonist GNTI $(1.5 \mu \mathrm{m} ; n=5$; Figures 1B,C). The membrane potential was at $-64.9 \pm 1.0 \mathrm{mV}$ in the presence of GNTI and at $-64.8 \pm 1.0 \mathrm{mV}$ in the presence of GNTI and DynA $\left(\Delta \mathrm{V}_{\mathrm{m}}=0.14 \pm 0.68 \mathrm{mV}\right)$. GNTI alone had no significant effect on the membrane potential of NPS neurons (One-Way analysis of variance [ANOVA]: $F_{(3,32)}=21.7$; post hoc test: GNTI vs. GNTI/DynA: $p=1$; baseline vs. DynA: $p=4.37 \mathrm{E}-6$; DynA vs. GNTI/DynA: $p=6.065 \mathrm{E}-6$; baseline vs. GNTI: $p=1$; DynA vs. GNTI: $p=7.368 \mathrm{E}-6$; Figures 1B,C). These data indicate that DynA hyperpolarizes NPS neurons in the periLC via activation of KORs. 
In a next set of experiments, the specific MOR and DOR agonists DAMGO and SNC-80, respectively, were bath-applied during current-clamp recordings. Neither $500 \mathrm{nM}$ DAMGO $\left(\mathrm{V}_{\mathrm{m}}=-63.1 \pm 1.3 \mathrm{mV}\right.$ vs. baseline $\mathrm{V}_{\mathrm{m}}=-63.4 \pm 1.4 \mathrm{mV}$; $\Delta \mathrm{V}_{\mathrm{m}}=-0.28 \pm 0.61 \mathrm{mV}, n=9 ; p=0.663 ; t=-0.453$; $\mathrm{DoF}=8)$, nor $500 \mathrm{nM}$ SNC-80 $\left(\mathrm{V}_{\mathrm{m}}=-63.7 \pm 1.4 \mathrm{mV}\right.$ vs. baseline $\mathrm{V}_{\mathrm{m}}=-63.7 \pm 1.0 \mathrm{mV} ; \Delta \mathrm{V}_{\mathrm{m}}=-0.06 \pm 0.77 \mathrm{mV} ; n=9 ; p=0.944$; $t=0.072$; DoF $=8$ ) changed the $\mathrm{V}_{\mathrm{m}}$ of NPS neurons (Figure 1D). The $\Delta \mathrm{V}_{\mathrm{m}}$ induced by DynA was significantly different from the $\Delta \mathrm{V}_{\mathrm{m}}$ induced by DAMGO or SNC-80 (One-way ANOVA $F_{(2,28)}=43.3$; $p=2.704 \mathrm{E}-9$; post hoc test: DynA vs. DAMGO: $p=3.593 \mathrm{E}-8$; DynA $v s$. SNC-80: $p=6.949 \mathrm{E}-8$; DAMGO vs. SNC-80: $p=1$; Figure 1D). These data indicate that NPS neurons at the periLC are responsive to KOR activation, but do not respond to MOR or DOR agonists.

As previously reported, NPS neurons in the periLC depolarize following activation of CRF1 (Jüngling et al., 2012). Since DynA and CRF can be coexpressed in LC afferents (Reyes et al., 2008; for review, see Van Bockstaele et al., 2010), we tested the effect of co-application of DynA and CRF (250 nM each) on the membrane potential of NPS neurons in the periLC. Under current-clamp conditions at a membrane potential of $-65 \mathrm{mV}(-64.1 \pm 0.9 \mathrm{mV})$ in presence of $1 \mu \mathrm{m}$ TTX, the co-application induced a hyperpolarization $(-69.9 \pm 3.3 \mathrm{mV})$ followed by a prominent depolarization $(-56.2 \pm 3.3 \mathrm{mV})$ upon wash (One-Way ANOVA: $F_{(2,12)}=33.06 ; p=1.313 \mathrm{E}-5$; post hoc test: baseline vs. DynA/CRF: $p=0.014$; baseline vs. wash: $p=0.002$; DynA/CRF vs. wash: $p=9.893 \mathrm{E}-6 ; n=5$; Figures $1 \mathrm{E}, \mathbf{F})$. Co-application of DynA and CRF in the presence of the CRF1 antagonist antalarmin $(4 \mu \mathrm{m})$ induced a more prominent and longer lasting hyperpolarization and abolished the depolarization during wash (One-Way ANOVA: $F_{(2,12)}=22.7$;post hoc test: baseline vs. DynA/CRF: $p=9.258 \mathrm{E}-5$; baseline vs. wash: $p=0.416$; DynA/CRF vs. wash: 0.001; $n=5$; Figures 1E,F). The DynA/CRF-induced hyperpolarization was significantly larger in presence of antalarmin $\left(\Delta \mathrm{V}_{\mathrm{m}}:-11.9 \pm 1.7 \mathrm{mV}\right)$ compared to recordings without antalarmin $\left(\Delta \mathrm{V}_{\mathrm{m}}:-5.9 \pm 1.2 \mathrm{mV}\right.$; unpaired $t$-test: $p=0.022 ; t=-2.84$; $\mathrm{DoF}=8)$. These findings indicate that the dynorphin and the CRF system interact at the level of single NPS neurons and induce a biphasic change in membrane potential in these cells.

In order to analyze the effect of DynA on active and passive membrane properties of NPS neurons, current-clamp recordings in the absence of TTX were done (Figures 2A,B). Hyper- and depolarizing current injections (500 $\mathrm{ms}$ duration; from -80 to $+100 \mathrm{pA} ; \Delta \mathrm{I}+10 \mathrm{pA}$ ) were delivered during current-clamp recordings at a membrane potential of $-60 \mathrm{mV}$ under baseline conditions and in presence of $250 \mathrm{nM}$ DynA (Figures 2A,B). In the presence of DynA, the shift of the membrane potential in response to hyperpolarizing currents was significantly less
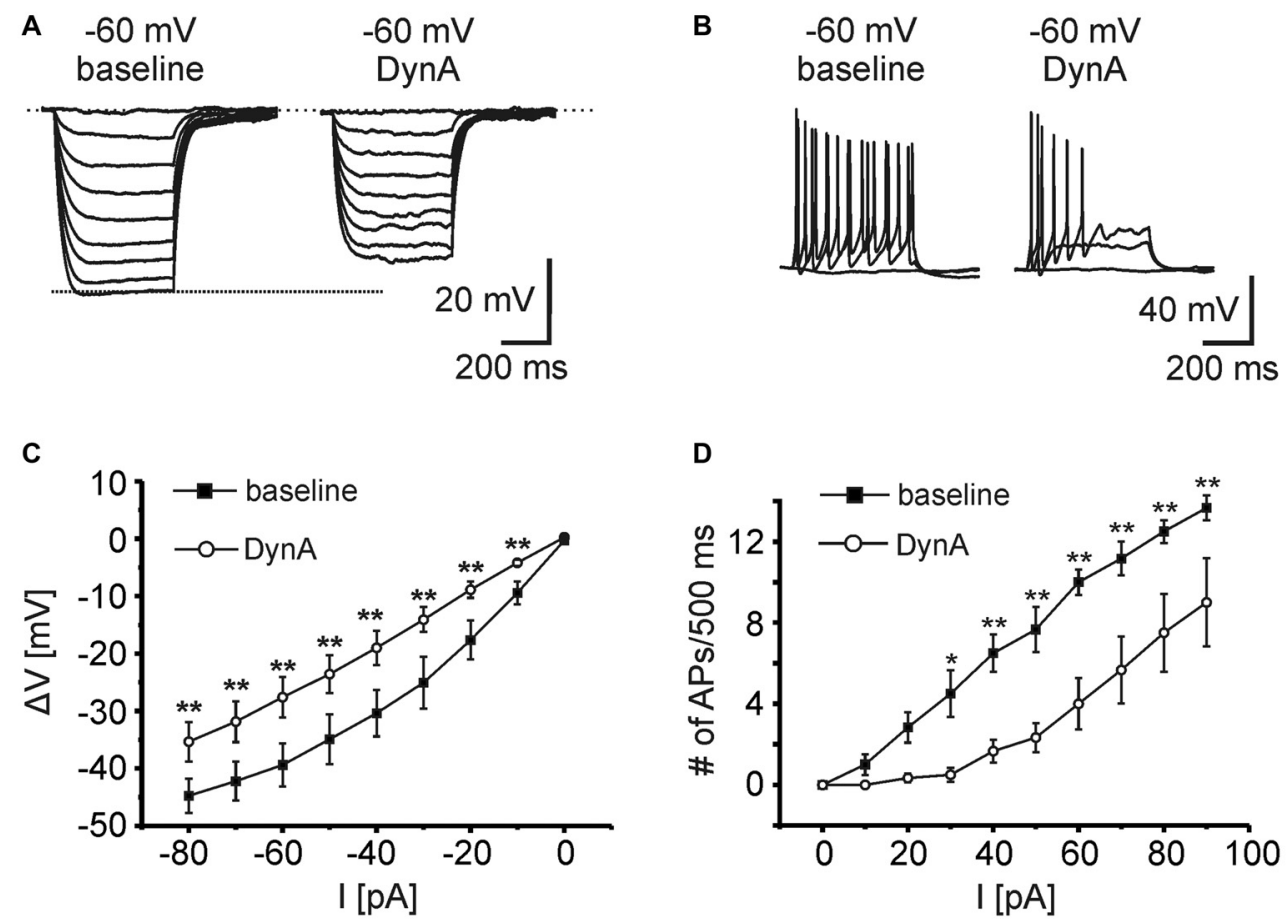

FIGURE 2 | KOR activation by DynA reduces the excitability of NPS neurons. (A) Passive membrane responses to hyperpolarizing current injections ( -80 to $0 \mathrm{pA} ; \Delta \mathrm{I}+10 \mathrm{pA} ; 500 \mathrm{~ms}$ duration) in absence and presence of $250 \mathrm{nM}$ DynA $(n=14)$. In presence of DynA, NPS neurons showed reduced voltage deflections in response to hyperpolarizing current injections. (B) Quantification of passive membrane responses to hyperpolarizing current injections. (C) Active membrane responses of NPS neurons to increasing depolarizing current injections (0-90 pA; $\Delta \mathrm{l}+10 \mathrm{pA} ; 500 \mathrm{~ms}$ duration). Application of $250 \mathrm{nM}$ DynA reduced the number of action potentials (APs) generated in response to the injected current. (D) Quantification of active membrane responses to depolarizing current injections. 
TABLE 1 | Summary of passive and active membrane properties recorded in the current-clamp mode at a membrane potential of $-60 \mathrm{mV}$ during baseline and in presence of DynA $(n=14)$.

\begin{tabular}{|c|c|c|c|c|c|}
\hline & \multicolumn{2}{|c|}{ Baseline } & \multicolumn{2}{|c|}{ DynA } & \multirow[b]{2}{*}{$p$-value } \\
\hline & Mean & SEM & Mean & SEM & \\
\hline $\mathrm{R}_{\text {in }}[\mathrm{M} \Omega]$ & 629 & 51 & 431 & 43 & 0.01 \\
\hline Tau [ms] & 38.2 & 1.9 & 32.7 & 3.3 & 0.17 \\
\hline Threshold [mV] & -41.2 & 0.6 & -38.9 & 1.0 & 0.06 \\
\hline AP amplitude [mV] & 73.7 & 0.7 & 68.4 & 0.9 & 0.03 \\
\hline $\mathrm{AHP}[\mathrm{mV}]$ & 17.6 & 0.6 & 18.8 & 0.5 & 0.15 \\
\hline Half-width [ms] & 1.2 & 0.1 & 1.1 & 0.1 & 0.31 \\
\hline slAHP [mV] & 3.1 & 0.5 & 1.4 & 0.3 & 0.01 \\
\hline Amplitude last $[\mathrm{mV}]$ & 53.7 & 2.2 & 53.3 & 2.8 & 0.92 \\
\hline AHPlast [mV] & 17.0 & 0.9 & 18.8 & 0.7 & 0.13 \\
\hline Inst. frequency $[\mathrm{Hz}]$ & 34.8 & 1.7 & 28.7 & 2.5 & 0.06 \\
\hline Time to $1 \mathrm{st}$ AP [ms] & 12.2 & 0.8 & 14.0 & 1.3 & 0.25 \\
\hline
\end{tabular}

Significant changes are marked in bold. Differences with a strong trend, but not reaching the $p<0.05$ significance criteria are marked in italic.

at all tested currents (Figures 2A,C). These data indicate that DynA induces a reduction in input resistance, which is in line with previous observations (Jüngling et al., 2015). Additionally, in the presence of DynA, NPS neurons generated fewer APs in response to depolarizing current injections, reaching significance for all tested current injections from $+30 \mathrm{pA}$ on (Two-Way ANOVA with repeated measurements: $F_{(9,252)}=10.04$; there is a significant difference between baseline and DynA: $p<$ 0.0001; levels of significance for the post hoc test are indicated in the Figure 2D). A summary of other active and passive membrane properties analyzed during baseline conditions and in presence of DynA is given in Table $\mathbf{1}(n=14)$. In summary, DynA reduces the input resistance and excitability of NPS neurons.

\section{lonic Mechanisms Underlying DynA-Induced Hyperpolarization}

In order to reveal the ionic mechanisms underlying the hyperpolarization of NPS neurons following KOR activation, whole-cell voltage-clamp recordings were performed at a holding potential of $-60 \mathrm{mV}$ in presence of DNQX, AP-5, GBZ, CGP55845, and TTX (Figure 3A). In voltage-clamp mode, $250 \mathrm{nM}$ DynA induced an outward current of $34.9 \pm 4.7 \mathrm{pA}$ $(n=5)$. The current amplitude was dose-dependent (50 nM: $11.5 \pm 4.6 \mathrm{pA}, n=4 ; 100 \mathrm{nM}: 12.6 \pm 2.6 \mathrm{pA}, n=3$; $200 \mathrm{nM}: 31.5 \pm 6.4 \mathrm{pA}, n=6 ; 250 \mathrm{nM}: 37.2 \pm 4.2 \mathrm{pA}, n=9$; 500 nM: $38.3 \pm 4.5 \mathrm{pA}, n=8$; Figure 4B). The dose-response curve indicates that $250 \mathrm{nM}$ DynA induced a near-maximal activation of KORs, since 500 nM DynA did not induce a larger current (Figure 3B).

To identify the DynA-induced current, a voltage-clamp ramp protocol ( -120 to $-20 \mathrm{mV}, 3 \mathrm{~s}, 60 \mathrm{~s}$ inter-trial interval) was applied during baseline conditions and in the presence of DynA (Figure 3C). The DynA-induced current was calculated by subtraction of the currents during baseline conditions from those recorded in the presence of DynA (Figure 3D), and current-voltage (I-V) relations were constructed. DynA induced an outward current that reversed at $-106.5 \pm 4.6 \mathrm{mV}$ $(n=5)$, a value close to the estimated potassium equilibrium potential for the present recording conditions $(-109 \mathrm{mV})$. In addition, the DynA-induced current displayed slight inward rectification. To test a possible effect of DynA on adenylyl cyclase-dependent pathways, $150 \mu \mathrm{m} 8$-Br-cAMP, which clamps the intracellular cAMP level at a high level, was included in the recording pipette during voltage-clamp recordings. The inclusion of 8-Br-cAMP reduced the DynA-induced outward current in NPS neurons without reaching statistical significance (DynA-induced current in control: $37.2 \pm 4.2 \mathrm{pA} ; n=9$; DynAinduced current in presence of 8-Br-cAMP: $19.6 \pm 6.3 ; n=4$; Figure $3 F$ ), suggesting that the observed current is mediated in parts via a down-regulation of AC activity. Furthermore, a cesium-methanesulfonate based intracellular solution (Csmet) abolished the DynA-induced current completely (DynAinduced current with Cs-met: $-6.7 \pm 3.5 \mathrm{pA} ; n=4$ ), providing further evidence for involvement of potassium conductances following KOR stimulation. Blocking ROMK1 and GIRK1/4 potassium channels by adding $200 \mathrm{nM}$ tertiapin $\mathrm{Q}$ reduced the DynA-induced current to $10.6 \pm 4.8 \mathrm{pA} \quad(n=8$; Figures 3E,F). The DynA-induced current was completely abolished in $0.5 \mu \mathrm{m} \mathrm{BaCl}$, which blocks a broad spectrum of inwardly rectifying potassium channels (DynA-induced current in $\mathrm{BaCl}_{2}:-2.1 \pm 5.9 \mathrm{pA}, n=6$; Figures 3E,F; One-Way ANOVA: $F_{(4,26)}=12.67 ; p=7.497 \mathrm{E}-6$; post hoc test: DynA control vs. $\mathrm{CsCl}_{2}: p=5.283 \mathrm{E}-5$; DynA control vs. tertiapin $\mathrm{Q}: p=0.002$; DynA control vs. $\left.\mathrm{BaCl}_{2}: p=3.907 \mathrm{E}-5\right)$. These data provide evidence that the DynA-induced current is mediated by inwardly rectifying potassium channels and that the majority of these channels are tertiapin Q-sensitive GIRK1/4 channels.

\section{DynA Negatively Modulates GABAergic Transmission}

In a next set of experiments, the AP-dependent release of GABA was investigated by analyzing electrically evoked IPSCs (eIPSCs) in NPS neurons. The stimulation electrode was positioned rostro-laterally to the NPS neuron cluster (Figure 4A). The eIPSCs appeared as inward currents and were sensitive to the $\mathrm{GABA}_{\mathrm{A}} \mathrm{R}$ antagonist GBZ $(n=3$; Figure 4B). The eIPSCs had a mean amplitude of $-122 \pm 31 \mathrm{pA}$ with a failure rate of $11.2 \pm 2.9 \%(n=11)$. Application of $250 \mathrm{nM}$ DynA reduced normalized mean eIPSC amplitudes to $0.53 \pm 0.07$. This effect was completely reversed upon wash out of DynA. At the end of the recording, the amplitude of eIPSCs recovered to $0.99 \pm 0.18$ of baseline (One-Way ANOVA: $F_{(2,30)}=0.008$; post hoc test: baseline vs. DynA: $p=0.019$; baseline vs. wash: $p=1$; wash vs. DynA: $p=0.019 ; n=11$; Figures 4C,D). The failure rate of eIPSCs increased from $11.5 \pm 2.7 \%$ during baseline conditions to $43.2 \pm 9.2 \%$ in presence of DynA and reversed to $12.5 \pm 4.2 \%$ after wash out of DynA (One-Way ANOVA: $F_{(2,29)}=8.67 ; p=0.001$; post hoc test: baseline vs. DynA: $p=0.003$; baseline vs. wash: $p=1$; wash vs. DynA: $p=0.005$; Figure 4D). Neither the mean latency of the eIPSCs (baseline: $2.2 \pm 0.2 \mathrm{~ms}$; DynA: $2.3 \pm 0.2 \mathrm{~ms}$ ), nor the eIPSC kinetics such as rise time $10-90 \%$ or decay time 
A

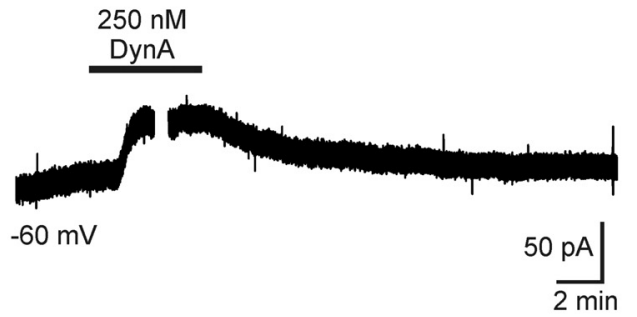

C

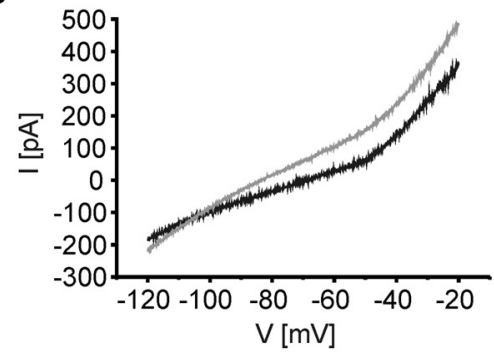

E
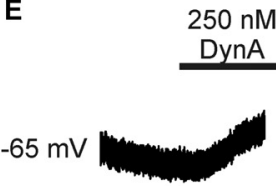

150 M 8-Br-cAMP

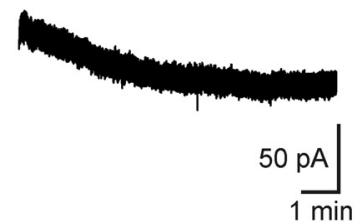

B

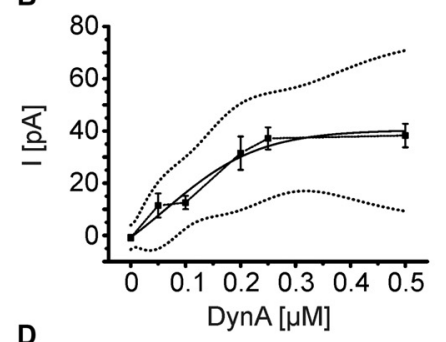

D

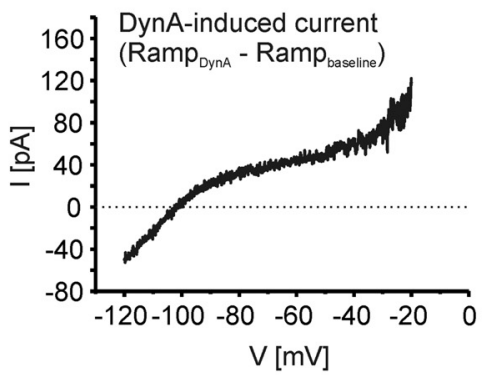

250 nM DynA

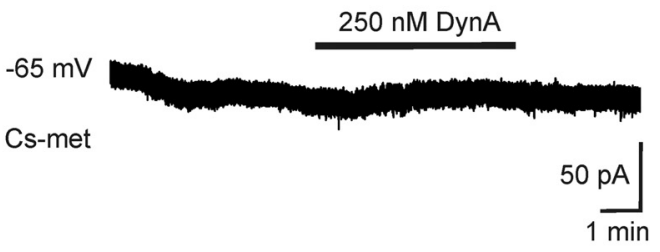

F
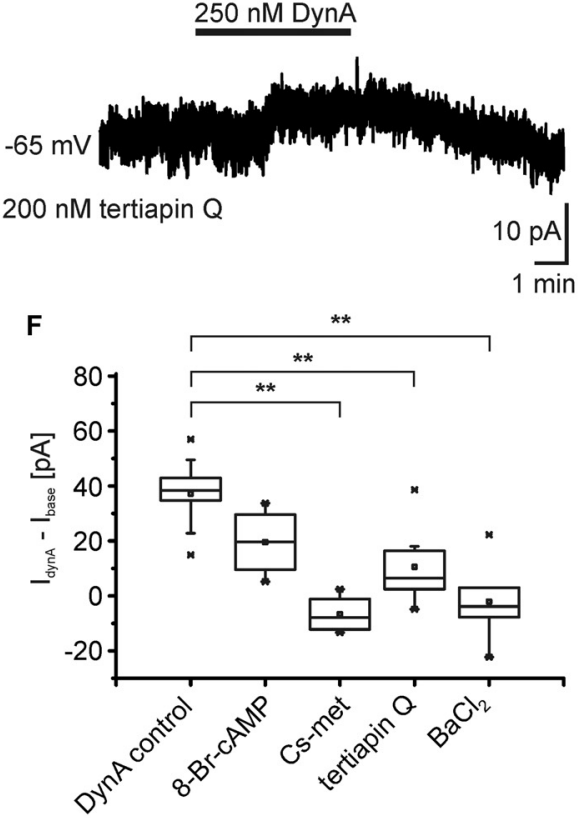

FIGURE 3 | lonic mechanisms underlying the DynA-induced hyperpolarization. (A) Example of NPS neuron recorded in the voltage-clamp mode at a holding potential of $-60 \mathrm{mV}$. Application of $250 \mathrm{nM}$ DynA induces a transient, outward directed current. The gap in the trace indicates the time point of a voltage-clamp ramp recording during DynA application. (B) Dose-response relationship of the DynA-induced outward current in response to $50 \mathrm{nM}(n=4), 100 \mathrm{nM}(n=3), 200 \mathrm{nM}$ $(n=6), 250 \mathrm{nM}(n=9)$ and $500 \mathrm{nM}(n=8)$ DynA. Dotted lines represent the upper and lower confidence interval (95\% level). (C) Sample voltage-clamp ramp recording (-120 mV to $-20 \mathrm{mV}, 3 \mathrm{~s}, 60 \mathrm{~s}$ inter-trial interval) during baseline (black) and in presence of DynA (gray). (D) Example of a DynA-induced current calculated from the voltage-clamp ramps shown in (C). The mean DynA-induced current reversed at $-106.5 \pm 4.6 \mathrm{mV}(n=5)$. (E) Example recordings of the DynA-induced current with inclusion of either 8-Br-cAMP or Cs-met in the internal recording solution or in presence of either $200 \mathrm{nM}$ tertiapin Q or $0.5 \mu \mathrm{m}$ BaCl 2 . (F) Quantification of the DynA-induced current during control conditions $(n=9)$, in the presence of 8-Br-cAMP $(n=4)$, Cs-met-sulfonate $(n=4)$, tertiapin Q $(n=8)$ or BaCl $2(n=6)$.

constant $\tau$ were significantly altered by DynA (rise time baseline: $1.2 \pm 0.1 \mathrm{~ms}$, DynA: $1.4 \pm 0.2 \mathrm{~ms}$; decay time constant baseline: $13.3 \pm 0.7 \mathrm{~ms}$, DynA: $14.6 \pm 0.9 \mathrm{~ms}$; Figure 4E). These data provide evidence that DynA negatively affects the efficacy of evoked GABAergic synaptic transmission onto NPS neurons.
To identify the possible site of action (pre- or postsynaptic) of the dynorphin system on GABAergic transmission, mIPSC in NPS neurons were recorded in voltage-clamp mode at a holdingpotential of $-65 \mathrm{mV}$ and in presence of $0.5 \mu \mathrm{m}$ TTX (Figure 5A). The mIPSC frequency was $2.5 \pm 0.2 \mathrm{~Hz}(n=8)$ during baseline recordings. The application of $250 \mathrm{nM}$ DynA induced a reduction 
A

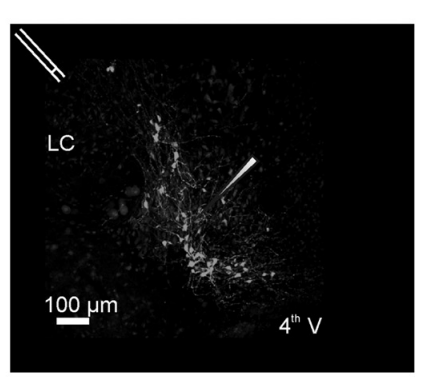

B

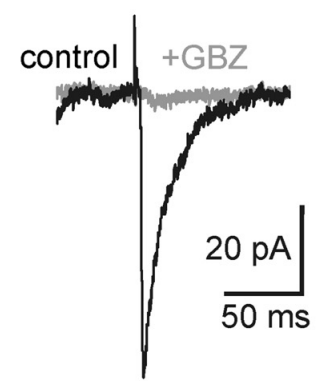

C

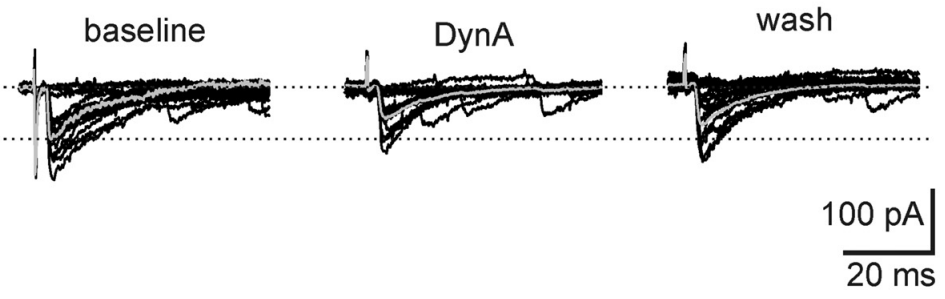

D
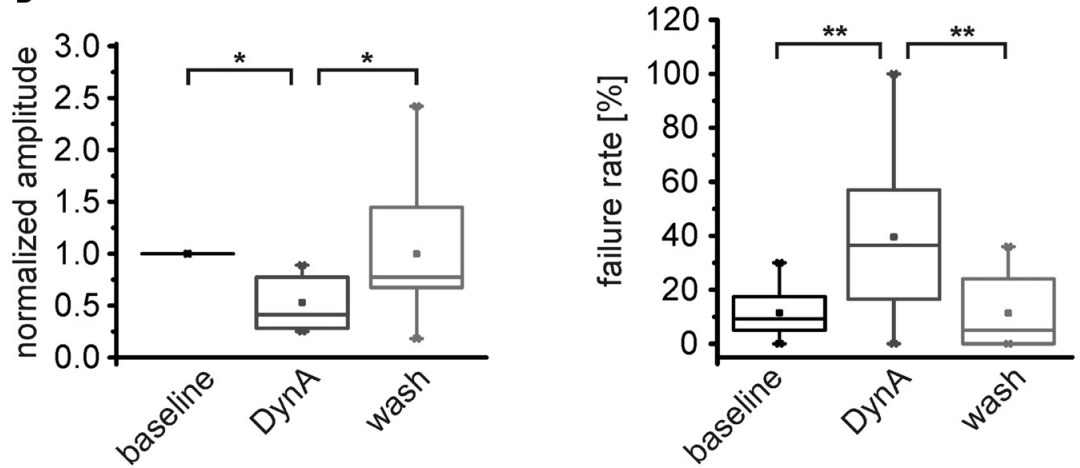

E
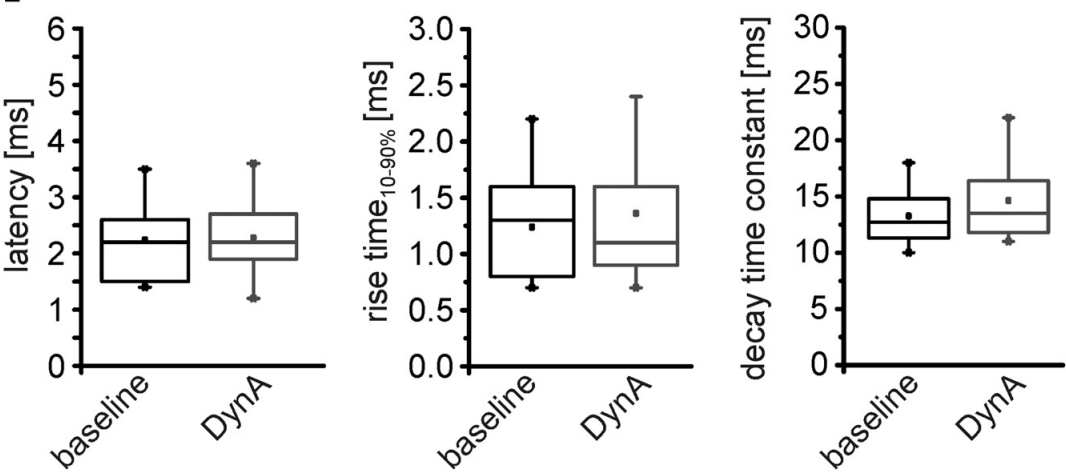

FIGURE 4 | DynA reduces the efficacy of GABAergic synapses on NPS neurons. (A) Scheme of the stimulation electrode position and recording site in a horizontal slice preparation of a transgenic nps-enhanced green fluorescent protein (EGFP) mouse. NPS neurons are located close to the fourth ventricle (4th $\mathrm{V}$ ) next to the locus coeruleus (LC). The stimulation electrode was placed rostrally to the recording site. (B) Evoked GABAergic postsynaptic responses (black) were sensitive to the $\mathrm{GABA}_{\mathrm{A}}$ receptor $(\mathrm{GABA} A \mathrm{R}$ ) antagonist gabazine (GBZ). (C) Examples of electrically evoked IPSCs (elPSCs) during baseline, in presence of 250 nM DynA and after wash. The mean response is presented in gray. (D) Quantification of the mean normalized success amplitudes and failure rate of the elPSCs $(n=11)$.

(E) Quantification of the eIPSC latency, rise time $10-90 \%$ and decay time constant during baseline and in presence of DynA.

in mIPSC frequency to $1.0 \pm 0.2 \mathrm{~Hz}$, which reversed to $1.6 \pm 0.2$ after wash (One-Way ANOVA: $F_{(2,21)}=15.8 ; p=6.485 \mathrm{E}-5$; post hoc test: baseline vs. DynA: $p=4.753 \mathrm{E}-5$; baseline vs. wash: $p=0.0071$; wash vs. DynA: $p=0.1395 ; n=8$; Figures 5A,B).
The mean amplitude of the mIPSCs during baseline recordings was $-101 \pm 8.8 \mathrm{pA}$. Application of DynA slightly reduced the amplitude to $-87 \pm 6.8 \mathrm{pA}$, which remained at $87.1 \pm 8 \mathrm{pA}$ after wash (One-Way ANOVA: $F_{(2,21)}=1.06 ; p=0.364$; Figure 5C). 


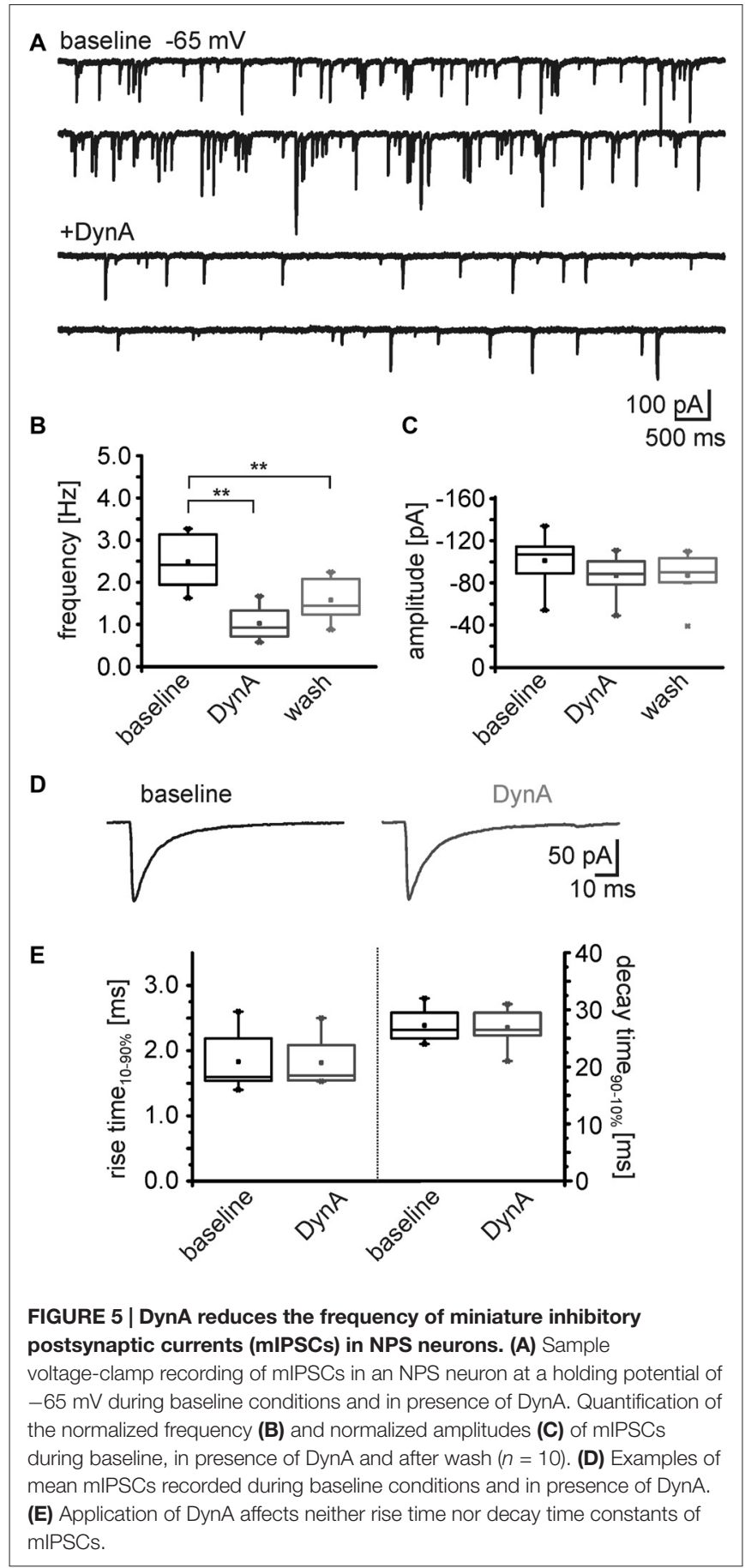

DynA did not affect the mIPSC kinetics (rise time $10-90 \%$ and

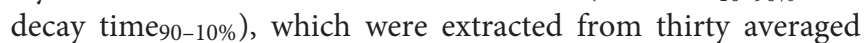
events during baseline and DynA application (Figure 5D). The rise time was $1.8 \pm 0.2 \mathrm{~ms}$ under control conditions and $1.8 \pm 0.2 \mathrm{~ms}$ in presence of DynA $(p=0.71 ; t=0.394 ; \operatorname{DoF}=7$; $n=8$ ), and the decay time $90-10 \%$ was $27.3 \pm 1.0 \mathrm{~ms}$ under control conditions and $26.9 \pm 1.2 \mathrm{~ms}$ in presence of DynA $(p=0.55$; $t=0.63$; DoF $=7 ; n=8$; Figure $5 \mathrm{E}$ ). The fact that DynA mainly affected the frequency of mIPSCs points to an involvement of presynaptic mechanisms.

\section{Differential Distribution of KOR1 and KOR2 Receptors}

At least two KOR subtypes, KOR1 and KOR2, can be differentiated pharmacologically (Zukin et al., 1988). Here, we used the KOR1 agonist U69,593 and the KOR2 agonist GR89696 (both at a concentration of $250 \mathrm{nM}$ ) to pharmacologically analyze the functional expression profile of these two receptor subtypes pre- and postsynaptically. During recordings of the membrane potential in presence of TTX, DNQX, CGP55845, GBZ and AP-5, U69,593 induced a significant hyperpolarization of NPS neurons (Figure 6A). The NPS neurons hyperpolarized from $-61 \pm 1 \mathrm{mV}$ during baseline recordings to $-67.4 \pm 0.6 \mathrm{mV}(p=9.815 \mathrm{E}$ $4 ; t=5.98$; DoF $=6 n=7$; Figure 6A). In contrast, GR89696 failed to induce a significant hyperpolarization (baseline: $-62.1 \pm 0.4 \mathrm{mV}$; GR89696: -63.0 $\pm 1.1 \mathrm{mV} ; p=0.33$; $t=1.02 ; \operatorname{DoF}=11 ; n=12 ;$ Figure 6A). The maximal shift of the membrane potential $\left(\Delta \mathrm{V}_{\text {membrane }}\right)$ was significantly larger upon U69,593 application $(-7.0 \pm 1.2 \mathrm{mV})$ compared to the $\Delta \mathrm{V}_{\text {membrane }}$ induced by $\mathrm{GR} 89696(-1.0 \pm 0.9 \mathrm{mV}$; $p=2.005 \mathrm{E}-4 ; t=-4.71 ; \mathrm{DoF}=17$; Figure 6B). These data indicate that NPS neurons express KOR1, but not KOR2 postsynaptically.

Conversely, both, the KOR1 and KOR2 agonists, modulated the frequency of mIPSCs recorded from NPS neurons in the periLC region (Figure 7A). The KOR1 agonist U69,593 reduced the mIPSC frequency from $2.3 \pm 0.3 \mathrm{~Hz}$ during baseline recordings to $1.2 \pm 0.3 \mathrm{~Hz}(1.4 \pm 0.3 \mathrm{~Hz}$ after wash; OneWay ANOVA: $F_{(2,21)}=4.14 ; p=0.031$; post hoc test: baseline vs. U69,593: $p=0.0397$; baseline vs. wash: $p=0.118$; wash vs. U69,593: $p=1 ; n=8$; Figure 7B). The mIPSC amplitude decreased from $-106 \pm 13 \mathrm{pA}$ to $-74 \pm 8 \mathrm{pA}$ in presence of U69,593 $(-81 \pm 7$ pA after wash; One-Way ANOVA: $F_{(2,21)}=3.28 ; p=0.058$; Figure 7C). Similarly, the KOR2 agonist GR89696 induced a decrease in frequency from $1.9 \pm 0.2 \mathrm{~Hz}$ during baseline to $1.1 \pm 0.2 \mathrm{~Hz}(1.3 \pm 0.2 \mathrm{~Hz}$ after wash; One-Way ANOVA: $F_{(2,18)}=4.84 ; p=0.021$; post hoc test: baseline vs. GR89696: $p=0.019$; baseline vs. wash: $p=0.25$; wash vs. GR89696: $p=0.68 ; n=7$; Figure 7B). The amplitudes shifted from $-115 \pm 14 \mathrm{pA}$ during baseline to $-98 \pm 13 \mathrm{pA}$ and $-102 \pm 14 \mathrm{pA}$ in presence of GR89696 and after wash, respectively (One-Way ANOVA: $F_{(2,18)}=0.95 ; p=0.4 ; n=7$; Figure 7C). In summary, both, KOR1 and KOR2 agonists, affect the frequency of mIPSCs, indicating a presynaptic mode of action of both pharmacologically distinct receptor subtypes.

\section{DISCUSSION}

The NPS system has been shown to be anxiolytic (Xu et al., 2004; Jüngling et al., 2008; Meis et al., 2008) and to attenuate stressinduced impairment of fear extinction (Chauveau et al., 2012). NPS neurons in the periLC depolarize in response to CRF application and subsequent CRF1 receptor activation (Jüngling et al., 2012). Moreover, stress exposure increases the expression 

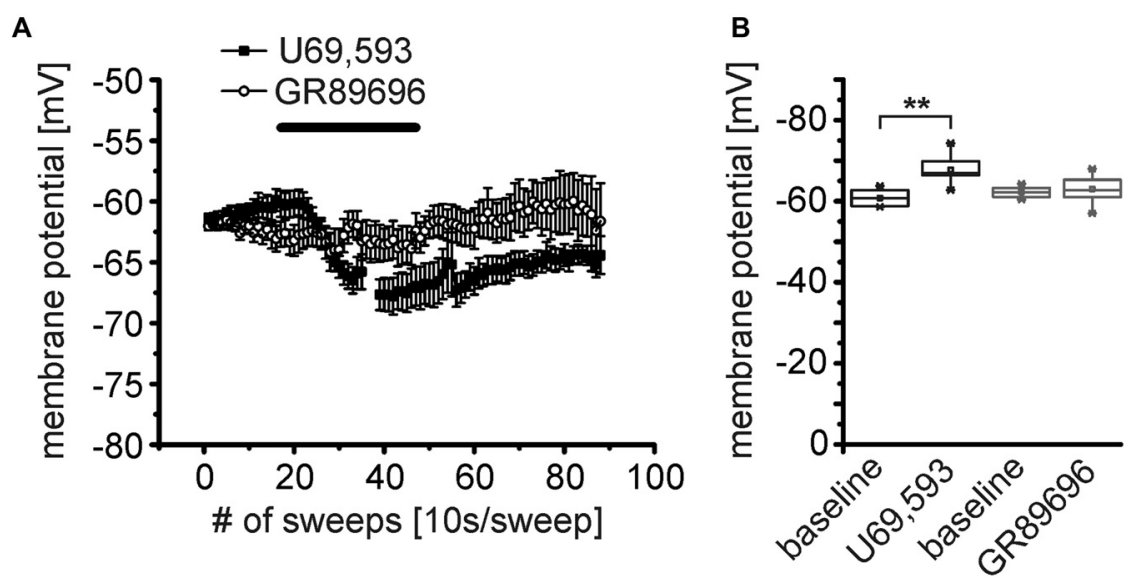

FIGURE 6 | The $\kappa$-opioid receptor 1 (KOR1) agonist U69,593 mimics the effect of DynA on the membrane potential of NPS neurons. (A) Effect of the KOR1 agonist U69,593 (250 nM; $n=7$ ) and the KOR2 agonist GR89696 (250 nM; $n=12$ ) on the membrane potential of NPS neurons. (B) Quantification of the effects of KOR1 and KOR2 agonists on the membrane potential. Note the lack of effect of the KOR2 agonist GR89696.
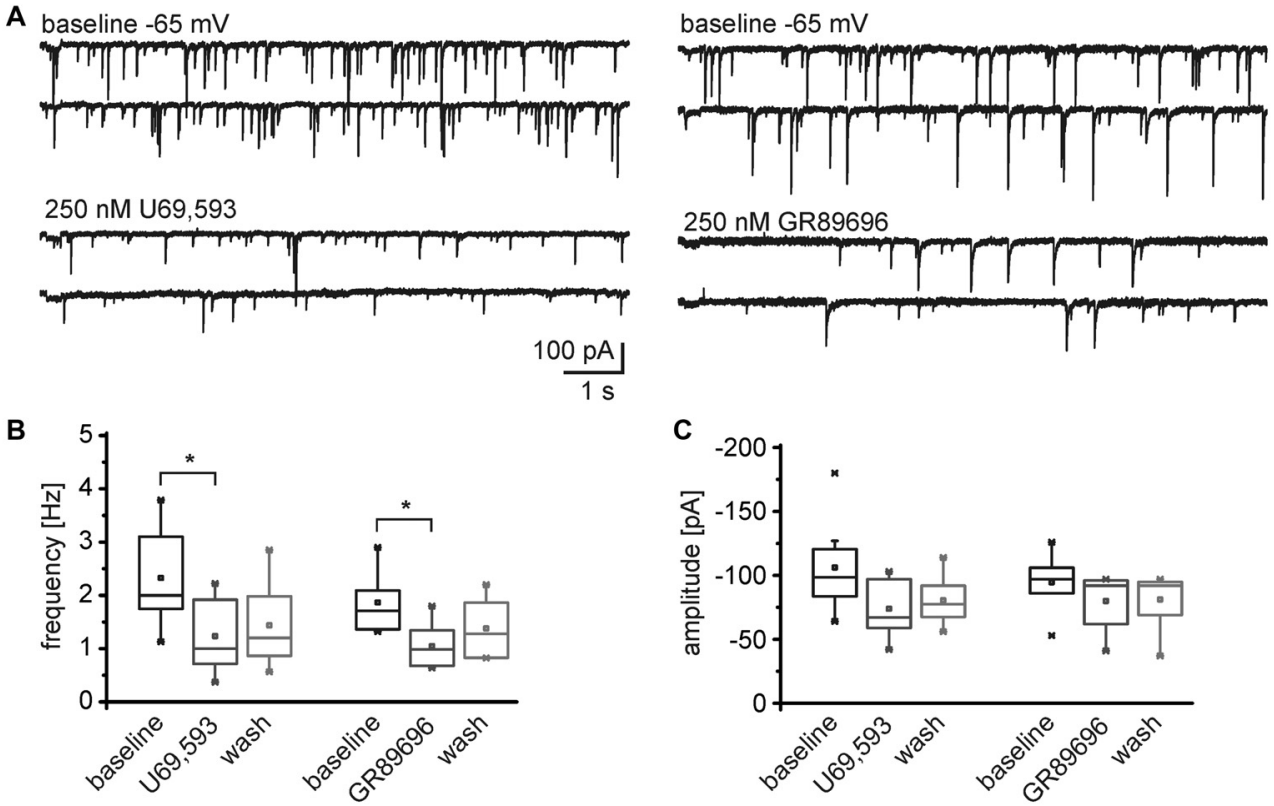

FIGURE 7 | Both, KOR1 and KOR2 agonists, reduce the frequency of mIPSCs in NPS neurons. (A) Examples of mIPSCs recorded during baseline conditions and in presence of U69,593 (left) or GR89696 (right) in the voltage-clamp mode at a holding potential of -65 mV. Quantification of the normalized frequency (B) and normalized amplitudes (C) during baseline conditions, in presence of U69,593 $(n=7)$ or GR89696 $(n=7)$, and after wash.

of the immediate early gene cfos in NPS neurons (Jüngling et al., 2012), and NPS levels are increased during stressful encounters in the basolateral amygdala in vivo (Ebner et al., 2011). These data strongly suggest that the NPS system is active during stressful experiences. Conversely, there is evidence that a variety of stress effects, such as aversion and depressive-like effects are mediated by the kappa opioid system (Mague et al., 2003; McLaughlin et al., 2003; Smith et al., 2012; Van Bockstaele et al., 2010). Additionally, interference with the kappa opioid system by KOR antagonists reduces anxiety-like behavior (Knoll et al., 2007, 2011; Wittmann et al., 2009).

While the gene expression profile of NPS neurons in the periLC indicates the presence of KORs as well as DORs and MORs (Liu et al., 2011), only the endogenous KOR ligand DynA but neither a specific MOR nor a specific DOR agonist induced a strong hyperpolarization of the membrane potential of NPS neurons. Using membrane potential as read out of receptor stimulation, these data indicate that, in contrast to 
KORs, MORs and DORs are not expressed postsynaptically in the NPS neurons. This conclusion does not exclude the possibility that MORs and DORs are functionally expressed at presynaptic sites.

The data presented here provide evidence that NPS neurons are inhibited following KOR activation. Conversely, NPS neurons are activated by the activation of CRF1 by either CRF or stressin I (Jüngling et al., 2012). Since DynA and CRF are co-expressed e.g., in LC projecting neurons of the central amygdala (Reyes et al., 2011), both substances might be coreleased. Co-application of DynA and CRF induced a biphasic response in NPS neurons, displaying a hyperpolarization followed by a depolarization upon wash. These data indicate that DynA is apt to attenuate CRF-induced activation of NPS neurons. Contrariwise, CRF diminishes the DynA-dependent hyperpolarization.

Application of DynA decreased the excitability and the input resistance of NPS neurons. Both effects seem to be mediated by an increase in the potassium conductance. It has previously been shown that KORs are intracellularly linked to inhibitory $\mathrm{G}_{\mathrm{i} / \mathrm{o}}$-proteins (Law et al., 2000), and activate inwardly rectifying potassium channels (GIRK; Henry et al., 1995). The DynA-induced outward current displayed rectifying properties and was sensitive to $\mathrm{Cs}^{+}$in the internal solution, strongly arguing for an activation of potassium conductances in NPS neurons in response to DynA. The inclusion of cAMP in the intracellular solution did not significantly alter the DynAinduced current, excluding a potential role of adenylyl cyclases which has been observed in other types of neurons (Attali et al., 1989; Lawrence and Bidlack, 1993). Moreover, in presence of barium, the DynA-induced current was abolished, arguing for a major contribution of inwardly rectifying potassium channels. Of the four $G$ protein-coupled inwardly rectifying potassium channel subunits (GIRK1-4) expressed in the rodent brain (Kobayashi et al., 1995; Karschin et al., 1996), the subunits GIRK1 and GIRK4 are sensitive to tertiapin Q, whereas GIRK2 and GIRK3 are insensitive (Jin et al., 1999). The DynA-induced current in NPS neurons was significantly reduced in presence of tertiapin Q, indicating the activation of GIRK1/4 downstream of the KOR. As seen in the box plots (Figure 3F), the DynAinduced currents were not abolished in all recorded neurons, pointing to the expression of tertiapin Q-insensitive GIRKs. In addition, $G$ protein signaling beyond the canonical seven transmembrane domain receptors and $G$ protein-independent pathways of these receptors may exist, including roles in receptor tyrosine kinase signaling and activity of G-protein accessory proteins (Sun et al., 2007; Coulon et al., 2010; Marty and Ye, 2010; Sato and Ishikawa, 2010).

Pharmacologically, two KORs, KOR1 and KOR2, can be distinguished by the use of specific agonists (Nock et al., 1988; Zukin et al., 1988; Rothman et al., 1989; Butelman et al., 2001). The KOR1-specific agonist U69,593, but not the KOR2 agonist GR89696, mimicked the hyperpolarizing effect of DynA in NPS neurons. A similar differential effect of KOR1 and KOR2 receptors has been described in proopiomelanocortin neurons (POMC) in the arcuate nucleus where KOR2 agonists but not KOR1 agonists induce a GIRK-dependent outward current
(Zhang and van den Pol, 2013). Taken together, DynA induces a hyperpolarization and decreases the excitability of NPS neurons via KOR1.

The kappa opioid system has been shown to negatively regulate the release of GABA from synapses in various neuronal circuits (Hjelmstad and Fields, 2001, 2003; Li et al., 2012). During local electrical stimulation of GABAergic inputs onto NPS neurons in the periLC, application of DynA induced a significant increase in the failure rate of the evoked release. Concomitantly, a clear trend towards a transiently decreased mean amplitude of evoked IPSCs was observed. In contrast, the kinetic of eIPSCs was largely unaffected. Moreover, DynA and the KOR1- and KOR2specific agonists, U69,593 and GR89696, respectively, reduced the frequency of GABAergic mIPSCs recorded in the presence of TTX. These data indicate that KORs can reduce GABA release by a presynaptic mode of action. From our data it cannot be concluded whether KOR1 and KOR2 receptors are expressed in the same or in separated synaptic terminals. It is known that there is formation of heterodimers of $\mathrm{G}$ protein-coupled receptors, which affects receptor pharmacology and signaling of the interacting subtypes (Prinster et al., 2005). Whether or not such an interaction of KOR1 and KOR2 plays a role in GABAergic afferents to NPS neurons in the periLC should be investigated in future studies.

It has previously been shown that the KOR1 agonist U69,593 attenuates GABA release by a reduction of the presynaptic $\mathrm{Ca}^{2+}$ influx via N-type channels (Hjelmstad and Fields, 2003). Effects of DynA on the frequency of GABAergic IPSCs have also been described for POMC and neuropeptide $\mathrm{Y}$ neurons in the arcuate nucleus (Zhang and van den Pol, 2013). Thus, it seems that mainly presynaptic mechanisms contribute to the observed reduction of the GABAergic synaptic transmission by KOR agonists. This would imply that KOR1 and KOR2 are present at presynaptic terminals of GABAergic synapses, whereas only KOR1 mediates the observed hyperpolarization via a direct postsynaptic mechanism. The activation of either presynaptic or postsynaptic located KORs could have differential effects on the excitability of NPS neurons. Activation of somatic KORs, inducing a prominent hyperpolarization, most likely would inactivate NPS neurons, and subsequently would cease the release of glutamate and NPS in the target regions of these neurons. Conversely, a predominant KOR activation on GABAergic terminals could mediate a disinhibition of NPS neurons due to a reduction of GABA release. Dynorphin could also act in an autoinhibitory mode since GABAergic afferents to NPS neurons in the periLC can contain dynorphin (Jüngling et al., 2015). In this scenario, heavily active GABAergic terminals control the amount of GABA release by co-releasing dynorphin. The net effect of KOR activation, either pre- and/or postsynaptically, on NPS neurons in the periLC in vivo remains elusive and demands further investigation.

Dynorphin-containing afferents can arise from various brain regions such as the hypothalamic paraventricular nucleus $(\mathrm{PVN}$; Watson et al., 1983; Reyes et al., 2005), the bed nucleus of the stria terminalis (BNST; Van Bockstaele et al., 1999b), the nucleus of the solitary tract (NTS; Van Bockstaele et al., 1999a) or the 
central nucleus of the amygdala (CeA; Reyes et al., 2008, 2011). These brain regions send afferents to neurons of the LC and are involved in stress responses. Recently, neurons of the CeA expressing prodynorphin and/or somatostatin have been shown to form GABAergic synapses onto NPS neurons in the periLC (Jüngling et al., 2015), bringing them into the ideal position to counteract the activity of NPS neurons during stress exposure or anxiety. Based on these findings, it is tempting to speculate that anxiolytic-like effects in rats upon KOR antagonism (Knoll et al., 2007) are influenced by a disinhibition of the NPS system and/or a more pronounced CRF effect on NPS neurons in absence of KOR activity. Since the NPS system is present in a variety of brain regions and modulates different cognitive and autonomic states, the interaction of the dynorphinergic and the NPS system can be manifold and demands further investigation. To date it is unclear during which conditions (relevant stressor or phasic vs. chronic stress) KORs in NPS neurons are activated. In conclusion, our data provide evidence indicating that the NPS and the kappa opioid system are interacting and that the endogenous opioid dynorphin is suited to inhibit NPS neurons at the LC. In turn, NPS release in putative target regions will decrease during opioid-mediated

\section{REFERENCES}

Attali, B., Saya, D., and Vogel, Z. (1989). $\kappa$-opiate agonists inhibit adenylate cyclase and produce heterologous desensitization in rat spinal cord. J. Neurochem. 52, 360-369. doi: 10.1111/j.1471-4159.1989.tb09130.x

Bruchas, M. R., Land, B. B., and Chavkin, C. (2007). The dynorphin/kappa opioid system as a modulator of stress-induced and pro-addictive behaviors. Brain Res. 1314, 44-55. doi: 10.1016/j.brainres.2009.08.062

Butelman, E. R., Ko, M. C., Traynor, J. R., Vivian, J. A., Kreek, M. J., and Woods, J. H. (2001). GR89,696: a potent $\kappa$-opioid agonist with subtype selectivity in rhesus monkeys. J. Pharmacol. Exp. Ther. 298, 1049-1059.

Carlezon, W. A., Beguin, C., DiNieri, J. A., Baumann, M. H., Richards, M. R., Todtenkopf, M. S., et al. (2006). Depressive-like effects of the $\kappa$-opioid receptor agonist salvinorin A on behavior and neurochemistry in rats. J. Pharmacol. Exp. Ther. 316, 440-447. doi: 10.1124/jpet.105.092304

Chauveau, F., Lange, M. D., Jungling, K., Lesting, J., Seidenbecher, T., and Pape, H. C. (2012). Prevention of stress-impaired fear extinction through neuropeptide s action in the lateral amygdala. Neuropsychopharmacology 37, 1588-1599. doi: 10.1038/npp.2012.3

Chavkin, C., James, I. F., and Goldstein, A. (1982). Dynorphin is a specific endogenous ligand of the к-opioid receptor. Science 215, 413-415. doi: 10. 1126/science.6120570

Coulon, P., Kanyshkova, T., Broicher, T., Munsch, T., Wettschureck, N., Seidenbecher, T., et al. (2010). Activity modes in thalamocortical relay neurons are modulated by $\mathrm{G}_{\mathrm{q}} / \mathrm{G}_{1} 1$ family G-proteins - serotonergic and glutamatergic signalling. Front. Cell Neurosci. 4:132. doi: 10.3389/fncel.2010.00132

Dimitrov, E. L., Yanagawa, Y., and Usdin, T. B. (2013). Forebrain GABAergic projections to locus coeruleus in mouse. J. Comp. Neurol. 521, 2373-2397. doi: $10.1002 /$ cne.23291

Ebner, K., Rjabokon, A., Pape, H. C., and Singewald, N. (2011). Increased in vivo release of neuropeptide $\mathrm{S}$ in the amygdala of freely moving rats after local depolarisation and emotional stress. Amino Acids 41, 991-996. doi: 10. 1007/s00726-011-1058-0

Grudt, T. J., and Williams, J. T. (1993). к-Opioid receptors also increase potassium conductance. Proc. Natl. Acad. Sci. U S A 90, 11429-11432. doi: 10.1073/pnas. 90.23.11429

Grudt, T. J., and Williams, J. T. (1995). Opioid receptors and the regulation of ion conductances. Rev. Neurosci. 6, 279-286. doi: 10.1515/revneuro.1995.6. 3.279 inactivation of NPS neurons, exerting a variety of physiological consequences such as increased anxiety or vulnerability to stress exposure.

\section{AUTHOR CONTRIBUTIONS}

KJ study design, performed experiments, writing of the manuscript, data analysis. PB: performed experiments, writing of the manuscript, data analysis. LG: performed experiments, writing of the manuscript. H-CP: study design, writing of the manuscript.

\section{ACKNOWLEDGMENTS}

Transgenic NPS-EGFP mice were a kind gift of Prof. Rainer Reinscheid (Univ. Irvine, USA). We thank Prof. Thomas Budde (University of Münster) for technical advice. The project was funded by the German research foundation (DFG; SFB-TR58, TPA03 to H-CP; TPA07 to KJ), the IZKF (Interdisciplinary Centre for Clinical Research, Münster; $\mathrm{PaHC} 3 / 003 / 10$ to $\mathrm{H}-\mathrm{CP}$ and KJ), a Novartis Fellowship to PB, a CiM Fellowship (DFG) to LG, and a Max Planck Research Award (to H-CP).

Henry, D. J., Grandy, D. K., Lester, H. A., Davidson, N., and Chavkin, C. (1995). $\kappa$-opioid receptors couple to inwardly rectifying potassium channels when coexpressed by Xenopus oocytes. Mol. Pharmacol. 47, 551-557. doi: 10. 1016/0304-3940(95)11289-9

Hjelmstad, G. O., and Fields, H. L. (2003). Kappa opioid receptor activation in the nucleus accumbens inhibits glutamate and GABA release through different mechanisms. J. Neurophysiol. 89, 2389-2395. doi: 10.1152/jn.01115. 2002

Hjelmstad, G. O., and Fields, H. L. (2001). Kappa opioid receptor inhibition of glutamatergic transmission in the nucleus accumbens shell. J. Neurophysiol. 85, 1153-1158.

Jin, W., Klem, A. M., Lewis, J. H., Lu, Z. (1999). Mechanisms of inward-rectifier $\mathrm{K}^{+}$channel inhibition by tertiapin-Q. Biochemistry 38, 14294-14301.

Jüngling, K., Lange, M. D., Szkudlarek, H. J., Lesting, J., Erdmann, F. S., Doengi, M., et al. (2015). Increased gabaergic efficacy of central amygdala projections to neuropeptide s neurons in the brainstem during fear memory retrieval. Neuropsychopharmacology 40, 2753-2763. doi: 10.1038/npp.2015.125

Jüngling, K., Liu, X., Lesting, J., Coulon, P., Sosulina, L., Reinscheid, R. K., et al. (2012). Activation of neuropeptide S-expressing neurons in the locus coeruleus by corticotropin-releasing factor. J. Physiol. Lond. 590, 3701-3717. doi: 10. 1113/jphysiol.2011.226423

Jüngling, K., Seidenbecher, T., Sosulina, L., Lesting, J., Sangha, S., Clark, S. D., et al (2008). Neuropeptide S-mediated control of fear expression and extinction: role of intercalated GABAergic neurons in the amygdala. Neuron 59, 298-310. doi: 10.1016/j.neuron.2008.07.002

Karschin, C., Dissmann, E., Stühmer, W., Karschin, A. (1996). IRK(1-3) and GIRK(1-4) inwardly rectifying $\mathrm{K}^{+}$channel mRNAs are differentially expressed in the adult rat brain. J. Neurosci. 16, 3559-3570. doi: 10.1007/978-1-40206754-9_6895

Knoll, A. T., and Carlezon, W. A. (2010). Dynorphin, stress and depression. Brain Res. 1314, 56-73. doi: 10.1016/j.brainres.2009.09.074

Knoll, A. T., Meloni, E. G., Thomas, J. B., Carroll, F. I., and Carlezon, W. A. (2007). Anxiolytic-like effects of kappa-opioid receptor antagonists in models of unlearned and learned fear in rats. J. Pharmacol. Exp. Ther. 323, 838-845. doi: 10.1124/jpet.107.127415

Knoll, A. T., Muschamp, J. W., Sillivan, S. E., Ferguson, D., Dietz, D. M., Meloni, E. G., et al. (2011). Kappa opioid receptor signaling in the basolateral amygdala regulates conditioned fear and anxiety in rats. Biol. Psychiatry 70, 425-433. doi: 10.1016/j.biopsych.2011.03.017 
Kobayashi, T., Ikeda, K., Ichikawa, T., Abe, S., Togashi, S., Kumanishi, T. (1995). Molecular cloning of a mouse G-protein-activated $\mathrm{K}^{+}$channel (mGIRK1) and distinct distributions of three GIRK (GIRK1, 2 and 3) mRNAs in mouse brain. Biochem. Biophys. Res. Commun. 208, 1166-1173.

Kreibich, A., Reyes, B. A., Curtis, A. L., Ecke, L., Chavkin, C., Van Bockstaele, E. J., et al. (2008). Presynaptic inhibition of diverse afferents to the locus ceruleus by kappa-opiate receptors: a novel mechanism for regulating the central norepinephrine system. J. Neurosci. 28, 6516-6525. doi: 10.1523/jneurosci. 0390-08.2008

Land, B. B., Bruchas, M. R., Lemos, J. C., Xu, M., Melief, E. J., and Chavkin, C. (2008). The dysphoric component of stress is encoded by activation of the dynorphin kappa-opioid system. J. Neurosci. 28, 407-414. doi: 10. 1523/jneurosci.4458-07.2008

Land, B. B., Bruchas, M. R., Schattauer, S., Giardino, W. J., Aita, M., Messinger, D., et al. (2009). Activation of the $\mathrm{\kappa}$-opioid receptor in the dorsal raphe nucleus mediates the aversive effects of stress and reinstates drug seeking. Proc. Natl. Acad. Sci. U S A 106, 19168-19173. doi: 10.1073/pnas.0910705106

Law, P. Y., Wong, Y. H., and Loh, H. H. (2000). Molecular mechanisms and regulation of opioid receptor signaling. Annu. Rev. Pharmacol. Toxicol. 40, 389-430. doi: 10.1146/annurev.pharmtox.40.1.389

Lawrence, D. M., and Bidlack, J. M. (1993). The kappa opioid receptor expressed on the mouse R1.1 thymoma cell line is coupled to adenylyl cyclase through a pertussis toxin-sensitive guanine nucleotide-binding regulatory protein. J. Pharmacol. Exp. Ther. 266, 1678-1683.

Li, C., Pleil, K. E., Stamatakis, A. M., Busan, S., Vong, L., Lowell, B. B., et al. (2012). Presynaptic inhibition of gamma-aminobutyric acid release in the bed nucleus of the stria terminalis by kappa opioid receptor signaling. Biol. Psychiatry 71, 725-732. doi: 10.1016/j.biopsych.2011.11.015

Liu, X., Zeng, J., Zhou, A., Theodorsson, E., Fahrenkrug, J., and Reinscheid, R. K. (2011). Molecular fingerprint of neuropeptide S-producing neurons in the mouse brain. J. Comp. Neurol. 519, 1847-1866. doi: 10.1002/cne.22603

Ma, G. H., Miller, R. J., Kuznetsov, A., and Philipson, L. H. (1995). kappaOpioid receptor activates an inwardly rectifying $\mathrm{K}^{+}$channel by a $\mathrm{G}$ proteinlinked mechanism: coexpression in Xenopus oocytes. Mol. Pharmacol. 47, 1035-1040.

Mague, S. D., Pliakas, A. M., Todtenkopf, M. S., Tomasiewicz, H. C., Zhang, Y., Stevens, W. C., et al. (2003). Antidepressant-like effects of $\kappa$-opioid receptor antagonists in the forced swim test in rats. J. Pharmacol. Exp. Ther. 305, 323-330. doi: 10.1124/jpet.102.046433

Marty, C., and Ye, R. D. (2010). Heterotrimeric G protein signaling outside the realm of seven transmembrane domain receptors. Mol. Pharmacol. 78, 12-18. doi: $10.1124 / \mathrm{mol} .110 .063453$

McDermott, C. M., and Schrader, L. A. (2011). Activation of kappa opioid receptors increases intrinsic excitability of dentate gyrus granule cells. J. Physiol. 589, 3517-3532. doi: 10.1113/jphysiol.2011.211623

McLaughlin, J. P., Marton-Popovici, M., and Chavkin, C. (2003). Kappa opioid receptor antagonism and prodynorphin gene disruption block stress-induced behavioral responses. J. Neurosci. 23, 5674-5683.

Meis, S., Bergado-Acosta, J. R., Yanagawa, Y., Obata, K., Stork, O., and Munsch, T. (2008). Identification of a neuropeptide $S$ responsive circuitry shaping amygdala activity via the endopiriform nucleus. PLoS One 3:e2695. doi: 10.1371/journal.pone. 0002695

Nock, B., Rajpara, A., O’Connor, L. H., and Cicero, T. J. (1988). Autoradiography of [3H]U-69593 binding sites in rat brain: evidence for kappa opioid receptor subtypes. Eur. J. Pharmacol. 154, 27-34. doi: 10.1016/0014-2999(88)90359-7

Okamura, N., and Reinscheid, R. K. (2007). Neuropeptide S: a novel modulator of stress and arousal. Stress 10, 221-226.

Okamura, N., Garau, C., Duangdao, D. M., Clark, S. D., Jungling, K., Pape, H. C., et al. (2011). Neuropeptide $S$ enhances memory during the consolidation phase and interacts with noradrenergic systems in the brain. Neuropsychopharmacology 36, 744-752. doi: 10.1038/npp.2010.207

Pan, Z. Z. (2003). Kappa-opioid receptor-mediated enhancement of the hyperpolarization-activated current $(\mathrm{I}(\mathrm{h}))$ through mobilization of intracellular calcium in rat nucleus raphe magnus. J. Physiol. 548, 765-775. doi: 10. 1113/jphysiol.2002.037622

Prinster, S. C., Hague, C., and Hall, R. A. (2005). Heterodimerization of Proteincoupled receptors: Specificity and functional significance. Pharm. Rev. 57, 289-298. doi: 10.1124/pr.57.3.1
Reyes, B. A., Carvalho, A. F., Vakharia, K., and Van Bockstaele, E. J. (2011). Amygdalar peptidergic circuits regulating noradrenergic locus coeruleus neurons: linking limbic and arousal centers. Exp. Neurol. 230, 96-105. doi: 10. 1016/j.expneurol.2011.04.001

Reyes, B. A., Drolet, G., and Van Bockstaele, E. J. (2008). Dynorphin and stressrelated peptides in rat locus coeruleus: contribution of amygdalar efferents. J. Comp. Neurol. 508, 663-675. doi: 10.1002/cne.21683

Reyes, B. A., Valentino, R. J., Xu, G., and Van Bockstaele, E. J. (2005). Hypothalamic projections to locus coeruleus neurons in rat brain. Eur. J. Neurosci. 22, 93-106. doi: 10.1111/j.1460-9568.2005.04197.x

Rothman, R. B., France, C. P., Bykov, V., De Costa, B. R., Jacobson, A. E. Woods, J. H., et al. (1989). Pharmacological activities of optically pure enantiomers of the kappa opioid agonist, U50,488 and its cis diastereomer: evidence for three kappa receptor subtypes. Eur. J. Pharmacol. 167, 345-353. doi: 10.1016/0014-2999(89)90443-3

Sato, M., and Ishikawa, Y. (2010). Accessory proteins for heterotrimeric G-protein: implication in the cardiovascular system. Pathophysiology 17, 89-99. doi: 10. 1016/j.pathophys.2009.03.011

Smith, J. S., Schindler, A. G., Martinelli, E., Gustin, R. M., Bruchas, M. R., and Chavkin, C. (2012). Stress-induced activation of the dynorphin/kappaopioid receptor system in the amygdala potentiates nicotine conditioned place preference. J. Neurosci. 32, 1488-1495. doi: 10.1523/jneurosci.298011.2012

Sun, Y., McGarrigle, D., and Huang, X. Y. (2007). When a G protein-coupled receptor does not couple to a $\mathrm{G}$ protein. Mol. Biosyst. 3, 849-854. doi: 10. 1039/b706343a

Van Bockstaele, E. J., Peoples, J., and Telegan, P. (1999a). Efferent projections of the nucleus of the solitary tract to peri-locus coeruleus dendrites in rat brain: Evidence for a monosynaptic pathway. J. Comp. Neurol. 412, 410-428. doi: 10. 1002/(sici)1096-9861(19990927)412:3410::aid-cne33.0.co;2-f

Van Bockstaele, E. J., Peoples, J., and Valentino, R. J. (1999b). A.E. Bennett Research Award. Anatomic basis for differential regulation of the rostrolateral peri-locus coeruleus region by limbic afferents. Biol. Psychiatry. 46, 1352-1363. doi: 10.1016/s0006-3223(99)00213-9

Van Bockstaele, E. J., Reyes, B. A., and Valentino, R. J. (2010). The locus coeruleus: A key nucleus where stress and opioids intersect to mediate vulnerability to opiate abuse. Brain Res. 1314, 162-174. doi: 10.1016/j.brainres.2009. 09.036

Watson, S. J., Khachaturian, H., Taylor, L., Fischli, W., Goldstein, A., and Akil, H. (1983). Pro-dynorphin peptides are found in the same neurons throughout rat brain: immunocytochemical study. Proc. Natl. Acad. Sci. U S A 80, 891-894.

Wittmann, W., Schunk, E., Rosskothen, I., Gaburro, S., Singewald, N., Herzog, H., et al. (2009). Prodynorphin-derived peptides are critical modulators of anxiety and regulate neurochemistry and corticosterone. Neuropsychopharmacology 34, 775-785. doi: $10.1038 /$ npp.2008.142

Xu, Y. L., Reinscheid, R. K., Huitron-Resendiz, S., Clark, S. D., Wang, Z., Lin, S. H. et al. (2004). Neuropeptide S: a neuropeptide promoting arousal and anxiolyticlike effects. Neuron 43, 487-497. doi: 10.1016/j.neuron.2004.08.005

Zhang, X., and van den Pol, A. N. (2013). Direct inhibition of arcuate proopiomelanocortin neurons: a potential mechanism for the orexigenic actions of dynorphin. J. Physiol. 591, 1731-1747. doi: 10.1113/jphysiol.2012. 248385

Zukin, R. S., Eghbali, M., Olive, D., Unterwald, E. M., and Tempel, A. (1988). Characterization and visualization of rat and guinea pig brain $\kappa$-opioid receptors: evidence for kappa 1 and kappa 2 opioid receptors. Proc. Natl. Acad. Sci. U S A 85, 4061-4065.

Conflict of Interest Statement: The authors declare that the research was conducted in the absence of any commercial or financial relationships that could be construed as a potential conflict of interest.

Copyright (๑ 2016 Jüngling, Blaesse, Goedecke and Pape. This is an open-access article distributed under the terms of the Creative Commons Attribution License (CC $B Y)$. The use, distribution and reproduction in other forums is permitted, provided the original author(s) or licensor are credited and that the original publication in this journal is cited, in accordance with accepted academic practice. No use, distribution or reproduction is permitted which does not comply with these terms. 